\title{
LETTER \\ Correcting Distortion of Views into Aquarium and Its Accuracy
}

\section{Yukio ISHIHARA $^{\dagger \mathrm{a})}$, Nonmember and Makio ISHIHARA ${ }^{\dagger \dagger \mathrm{b})}$, Member}

\begin{abstract}
SUMMARY We present a way to correct light distortion of views looking into an aquarium. When we see fish in an aquarium, they appear closer and distorted due to light distortion. In order to correct the distortion, light rays travelling in the aquarium directly towards an observer should hit him/her after emerging from the aquarium. In this manuscript, those light rays are captured by a perspective camera at specific positions, not the observer position. And then it is shown that the taken images are successfully merged as a single one that is not affected by light distortion.

key words: distortion correction, aquarium, light distortion
\end{abstract}

\section{Introduction}

Do you have any experience of seeing fish distorted while you watch them in an aquarium, especially at a diagonal position to the surface? Those fish appear closer and distorted due to light distortion. Technically, light distortion stems from light refraction, where light rays bend passing through the surface of an aquarium. In this manuscript, we present a way to correct light distortion of views looking into an aquarium and construct novel views that are not affected by light distortion, or distortion-free views. Some studies dealt with light distortion for measurement of underwater objects [1], [2] and for 3D structure estimation [3], [4]. Construction of distortion-free views is however outside the scope of these studies. So far, no studies are found attempting to construct distortion-free views. These views could be constructed by the 3D structure estimation studied in [3], [4], but it seems inappropriate to create underwater atmosphere including drifting dust, ascending air bubbles, tiny creatures and the like. These surroundings help to perceive fish in depth. Therefore we use the image based rendering [5] to construct distortion-free views.

\section{Correction of Light Distortion}

Figure 1 illustrates how light distortion is corrected. An observer stands at $D \mathrm{~mm}$ to the glass. Rayl shows a straight line between the observer and a fish, but the observer doesn't see the fish in that direction because of light distortion. Ray2 needs to be seen at their position as Ray1 that comes from

Manuscript received May 9, 2014.

Manuscript publicized June 25, 2014.

${ }^{\dagger}$ The author is with Kyushu University, Fukuoka-shi, 8128582 Japan.

${ }^{\dagger}$ The author is with Fukuoka Institute of Technology, Fukuokashi, 811-0295 Japan.

a)E-mail: iyukio@ redoxnavi.med.kyushu-u.ac.jp

b) E-mail: m-ishihara@ fit.ac.jp

DOI: 10.1587/transinf.2014EDL8092

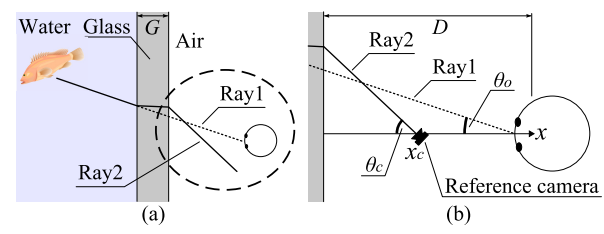

Fig. 1 Our approach to correct light distortion (top view). (a) Two key light rays. Ray1 travels without consideration of light refraction while Ray2 is refracted on the two boundaries: water to glass, glass to air. (b) The close-up of (a). Ray2 is captured by a reference camera.

$\theta_{o}$ off-center. First, Ray 2 is captured by a perspective camera at $x_{c}$ in the direction of $\theta_{c}$. This perspective camera is referred to as a reference camera. $x_{c}$ and $\theta_{c}$ are given by

$$
\begin{aligned}
& x_{c}=\frac{(G+D) \tan \theta_{o}-G \tan \theta^{\prime}}{\tan \theta_{c}}, \theta^{\prime}=\arcsin \left(\frac{n_{3}}{n_{2}} \sin \theta_{o}\right), \\
& \theta_{c}=\arcsin \left(\frac{n_{3}}{n_{1}} \sin \theta_{o}\right)
\end{aligned}
$$

where $n_{1}, n_{2}$ and $n_{3}$ are refractive indices for air (1.0), glass (1.52) and water (1.33), respectively. $G$ is the thickness of the glass aquarium. Next, Ray2 is mapped to Ray1 by Eqs. (1) and (2). Finally a pixel on the perspective image of the observer view, which corresponds to Ray1, is drawn in the color of Ray2. To draw the other pixels, the reference camera is placed at a series of positions on $\mathrm{x}$-axis and the process mentioned above is repeated to construct the observer view that is distortion-free.

Equations (1) and (2), the relationship between the reference camera and the observer in position and orientation, could be expressed by the pixel-wise varifocal camera model presented in the studies [2], [4]. The difference from these studies is that Ray1 has to cross $\mathrm{X}$-axis at the position of the observer, so most of Rays captured by the reference camera are unnecessary, but only Ray2 is used and mapped to Ray1. This is why the reference camera needs to move on the straight line of $\mathrm{x}$-axis and takes images for Ray1 coming to the observer from the other directions.

Our contribution is to provide positions of a reference camera in order to capture images necessary for construction of distortion-free observer views.

\section{Experiment}

Figure 2 shows a glass aquarium in an experimental setup. The thickness of the glass is $G=5 \mathrm{~mm}$ and the distance to the glass from an observer is $D=50 \mathrm{~mm}$. A reference cam- 


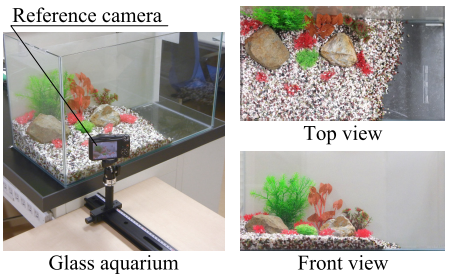

Fig. 2 A glass aquarium in an experimental setup. There is no water in the aquarium to see the right positions of water plants and rocks inside.

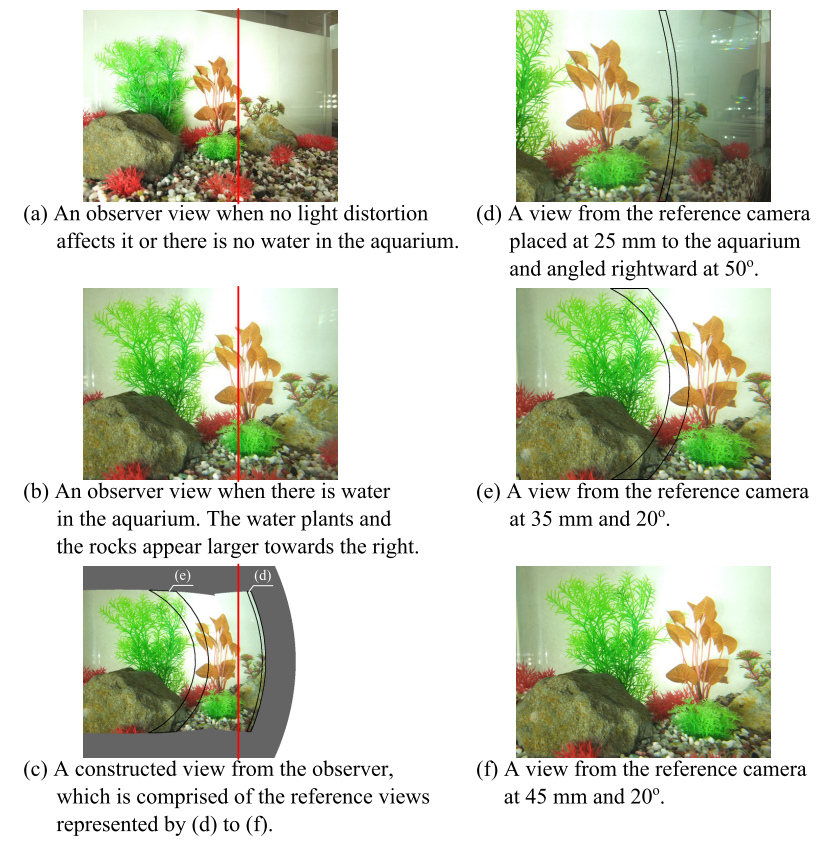

Fig. 3 Views from the observer and the reference camera in a real world environment. The vertical lines help understand to what extent the view is distorted and corrected.

era was moved by $1 \mathrm{~mm}$ from 25 to $50 \mathrm{~mm}$ to the glass, also angled rightward at $50^{\circ}$ for 25 to $31 \mathrm{~mm}$ and at $20^{\circ}$ for 32 to $50 \mathrm{~mm}$. At each position one reference view was taken, therefore 26 reference views in all were obtained. Another camera representing the observer was placed at $50 \mathrm{~mm}$ to the glass and angled rightward at $25^{\circ}$.

Figure 3 (a) shows a view from the observer who looks into the aquarium filled with no water. Figure 3 (b) shows the same view but the aquarium is filled with water. As expected, the view is distorted compared to (a). That is, objects in (b) appear larger towards the right, or the amount of light distortion increases as it goes right, which is consistent with the study [6]. Figure 3 (d) to (f) represent three of the reference views. Note that these views are all affected by light distortion. Finally as shown in Fig. 3 (c), the desired view from the observer was constructed from the reference views. Specifically, for example, pixels surrounded by a line in (d) and (e) contributed to the corresponding part of the view (c).

It should be mentioned that some peripheral part in Fig. 3 (c) is left grey. The grey could be drawn if reference views at 1 to $24 \mathrm{~mm}$ positions were available as well as up-

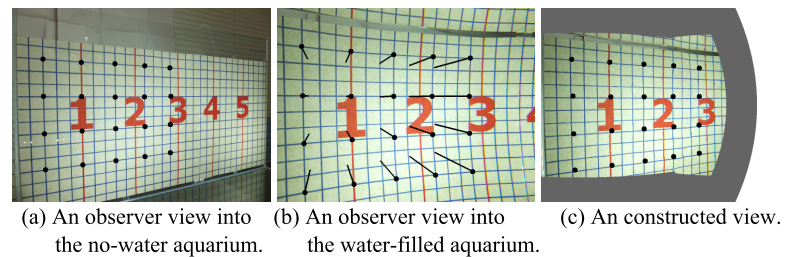

Fig. 4 Accuracy of constructed views. Twenty sampled points are shown in (a). The corresponding points and the connecting lines to those in (a) are shown in (b) and (c).

ward/downward ones. In this experiment, only rightward reference views were used for simplicity. And reference views up to $24 \mathrm{~mm}$ position were unable to be obtained because of no room to place the reference camera even closer to the glass.

In addition we show the accuracy of constructed views. A structured image of $2 \times 2 \mathrm{~cm}$ square lattice was placed on the back surface of the aquarium as shown in Fig. 4 (a). Twenty points were sampled at the intersections on this structured image. The corresponding points are shown in Fig. 4 (b) and (c). Connecting lines between the sampled and the corresponding points are also shown in the figures. The lengths of the lines in (b) and (c) are 83.07 and 1.74 pixels in average, respectively. 10 pixel length is equal to the radius of dots showing the sampled points. As a result, the constructed view (c) accurately shows the structured image without light distortion.

\section{Conclusions}

In this manuscript, we discussed a way to correct light distortion of views looking into an aquarium. An experiment in a real world environment confirmed that distortion-free observer views were successfully constructed from multiple reference views.

\section{References}

[1] T. Treibitz, Y. Schechner, C. Kunz, and H. Singh, Flat Refractive Geometry, IEEE Trans. Pattern Anal. Mach. Intell., vol.34, no.1, pp.5165, 2012.

[2] R. Kawahara, S. Nobuhara, and T. Matsuyama, "A pixel-wise varifocal camera model for efficient forward projection and linear extrinsic calibration of underwater cameras with flat housings," Proc. 2013 IEEE International Conference on Computer Vision Workshops, pp.819-824, 2013.

[3] L. Kang, L. Wu, and Y.-H. Yang, "Two-view underwater structure and motion for cameras under flat refractive interfaces," A. Fitzgibbon, S. Lazebnik, P. Perona, Y. Sato, C. Schmid (eds.) ECCV 2012, Part IV, LNCS, vol.7575, pp.303-316, Springer, Heidelberg, 2012.

[4] A. Sedlazeck and R. Koch, "Refractive structure-from-motion on underwater images," Proc. 2013 IEEE International Conference on Computer Vision, pp.57-64, 2013.

[5] S.J. Gortler, R. Grzeszczuk, R. Szeliski, and M.F. Cohen, "The lumigraph," Proc. SIGGRAPH 1996, pp.43-54, 1996.

[6] A. Agrawal, S. Ramalingam, Y. Taguchi, and V. Chari, "A theory of multi-layer flat refractive geometry," Proc. 2012 IEEE Conference on Computer Vision and Pattern Recognition, pp.3346-3353, 2012. 